\title{
ANÁLISE DA OCORRÊNCIA DE PARASITOS COM POTENCIAL ZOONÓTICO NO SOLO DE ESCOLAS MUNICIPAIS INFANTIS EM DOIS MUNICÍPIOS DO RIO GRANDE DO SUL
}

\author{
ANALYSIS OF THE OCCURRENCE OF PARASITES WITH \\ ZOONOTIC POTENTIAL IN THE SOIL OF MUNICIPAL \\ NURSERY SCHOOLS IN TWO MUNICIPALITIES IN RIO \\ GRANDE DO SUL STATE
}

\author{
Aline dos Santos Padilha ${ }^{1}$, Rita Leal Sperotto ${ }^{2}$, \\ Amanda Felipe Portella², Aline Henn²
}

\section{RESUMO}

Introdução: A contaminação de praças ou ambientes de recreação por ovos de geohelmintos constitui um problema de saúde pública. O solo, com relação aos helmintos parasitas se comporta como um hospedeiro intermediário. Recebe fezes ou água contaminada por parasitas em estágios não infectantes, oferecendo-lhes condições para o desenvolvimento e protege os parasitas em estágios infectantes durante certo tempo para, posteriormente, transmiti-lo ao homem. O objetivo é verificar a ocorrência e a contaminação ambiental por parasitas com potencial zoonótico no solo de escolas infantis em dois municípios do Rio Grande do Sul.

Métodos: Trata-se de um estudo delineamento transversal, observacional e descritivo. A coleta foi através de amostras de areia em dez escolas de dois municípios, as coletas do material foram de cinco pontos diferentes, tanto da profundidade quanto da superfície, totalizando-se 100 amostras analisadas. As amostras de areia foram processadas em laboratório através dos métodos de Rugai e Hoffman, Pons e Janer.

Resultados: Análise total $(\mathrm{N}=100)$ dos dois municípios, totalizando 50 amostras de cada. A contaminação das areias, por ovos de Ancylostoma spp., foi de $54 \%(n=27)$ no município I, sendo encontrados $56 \%(n=28)$ na cidade II.

Conclusão: A ocorrência de ovos de Ancylostoma spp. no ambiente de recreação enfatiza a importância da adoção de medidas restritivas rígidas que impeçam a entrada de animais, como cães e gatos em locais de lazer. Além desses parasitas serem capazes de comprometer a saúde de humanos e infectar outros animais.

Palavras-chave: Helmintíase; larva migrans; parasitoses; Ancylostoma spp; escola infantil

\begin{abstract}
Introduction: Contamination of squares or recreational environments with geohelminth eggs is a public health problem. The soil serves as an intermediate host to helminths. It receives feces or water contaminated with parasites in non-infective stages, providing them with conditions for development, and protects parasites in infective stages for some time and then transmits them to humans. The aim was to investigate the occurrence of environmental contamination with parasites with zoonotic potential in the soil of nursery schools in two municipalities of Rio Grande do Sul state.
\end{abstract}

Methods: A cross-sectional, observational and descriptive study was conducted. Soil samples were collected at ten schools in two municipalities, from five different
Clin Biomed Res. 2019;39(1):9-14

1 Centro de Ciências da Saúde e Agrárias, Universidade Federal de Santa Maria (UFSM). Santa Maria, RS, Brasil.

2 Centro de Ciências da Saúde e Agrárias, Universidade de Cruz Alta (UNICRUZ). Cruz Alta, RS, Brasil.

Autor correspondente: Rita Leal Sperotto rleal@unicruz.edu.br Centro de Ciências da Saúde e Agrárias, Universidade de Cruz Alta (UNICRUZ) Rodovia Municipal Jacob Della Méa, s/n. 98020-290, Cruz Alta, RS, Brasil. 
school sites in terms of both depth and surface. Soil samples were processed at a laboratory using the methods of Rugai and Hoffman, Pons and Janer.

Results: In total, 100 samples from the two municipalities were analyzed, 50 samples each. Soil contamination with Ancylostoma spp. eggs was $54 \%(n=27)$ in city I and $56 \%(n=28)$ in city II.

Conclusions: The occurrence of Ancylostoma spp. eggs in recreational environments shows the importance of adopting strict restrictive measures to prevent animals, such as dogs and cats, from entering leisure areas. Also, these parasites may compromise human health and infect other animals.

Keywords: Helminthiasis; larva migrans; parasitic diseases; Ancylostoma spp; nursery school

Cada vez a sociedade está mais igualitária, a mulher tem assumido vários papéis, sendo assim o primeiro contato de crianças com o ambiente externo cada vez mais cedo, na infância é fase de maior vulnerabilidade para o desenvolvimento de parasitoses, cujos os hábitos geofágicos ocasiona a ingestão de ovos embrionados de parasitas ou também pela penetração cutânea de larvas presentes no ambiente ${ }^{1,2}$. O solo exerce vantagem para várias espécies e formas biológicas de parasitos, nos ambientes como praças e parques, há trânsito livre de cães e gatos, os quais contaminam estes ambientes com suas fezes, eliminando até 15.000 ovos de parasitas por grama de material fecal, estes ovos permanecem viáveis por longo período no ambiente expondo a população humana ao risco de infecção, afetar o equilíbrio nutricional, gerar complicações no metabolismo normal intestinal e desenvolvimento de doenças, constituindo uma via de transmissão de zoonoses parasitárias ${ }^{2,3}$.

Em meio às espécies de helmintos encontrados no solo que os principais que causam danos ao homem são: Ancylostoma sp, Ascaris lumbricoides, Enterobius vermicularis, Trichuri $\mathrm{sp}$, Strongyloides stercoralis. Já entre os protozoários conta a presença da Giardia lamblia e o gênero Entamoeba ${ }^{4,5}$. Todavia, compreender a presença de parasitas com potencial zoonótico nestes locais, para que sejam tomadas medidas preventivas para controle de doenças e a interrupção do ciclo e da transmissão que poderá controlar zoonoses parasitárias.

A alta prevalência destas parasitoses na maioria das vezes, estar associada com as condições socioeconômicas, sanitárias e educacionais da sociedade. A contaminação da água, solo e alimentos através de parasitas torna-se fácil à dispersão de ovos, cistos ou larvas fazendo com que desencadeei as patologias relacionadas a cada parasita ${ }^{2,6}$. Dentre esses, há os Ancilostomídeos que considerados nematódeos pertencentes a família a Ancylostomatidae, apresentam nítido dimorfismo sexual e duas estruturas bastante características: cápsula bucal, nas fêmeas e machos e bolsa copuladora apenas em machos ${ }^{2}$. Esses parasitos têm como hospedeiros definitivos canídeos, felídeos e humanos, dependendo da espécie parasita. As infecções humanas por Ancilostomídeos ocorrem pela penetração e migração cutânea de larvas, principalmente, Ancylostoma braziliense e Ancylostoma caninum, os quais são encontrados no intestino delgado de cães e gatos. Larva Migrans Visceral (LMV), é ocasionada pela ingestão acidental de ovos infectantes, principalmente, de Toxocara canis, parasito intestinal de cães ${ }^{2,3,6}$.

As parasitoses constituem um sério problema de saúde pública, devido à alta prevalência de formas parasitárias viáveis em locais de fácil acesso humano e onde existam condições ambientais favoráveis à sua manutenção, sendo assim, a temperatura e a umidade elevadas favorecem o desenvolvimento das larvas até o estádio infectante , $^{5-9}$. Com isso, as informações obtidas através deste estudo poderão auxiliar em campanhas de prevenção a determinadas doenças parasitárias, além da tentativa de persuadir as autoridades locais a criar estratégias para inibir o trânsito de animais em locais públicos, como nos pátios das escolas.

Este tipo de estudo é justificado pela necessidade de constatar a presença de parasitas em locais habitados por indivíduos considerados mais suscetíveis à parasitoses, como as crianças, tendo em vista que outros estudos semelhantes foram desenvolvidos em várias regiões do Brasil, apontando a contaminação dos solos, o que é considerado de alto risco para saúde pública ${ }^{4,6,10,11}$. O presente trabalho tem como objetivo verificar a contaminação, por parasitos com potencial zoonótico, do solo das escolas infantis de dois municípios de regiões distintas do Estado do Rio Grande do Sul. 


\section{MÉTODOS}

O presente estudo apresenta um delineamento transversal, observacional e descritivo. O projeto inicial foi submetido ao Comitê de Ética em Pesquisa (CEP) da Universidade de Cruz Alta obtendo aprovação através do Parecer Consubstanciado: 2.510.800. O estudo englobou locais escolares que apresentavam áreas para recreação de crianças apresentando parquinho ou coleções de areia, assim através desta pesquisa buscou-se verificar a ocorrência de helmintos e protozoários em areia de escolas infantis municipais, além de observar os fatores externos, como a presença de lixo no pátio, presença de animais, umidade e irradiação solar, os quais poderiam justificar a positividade ou a negatividade das amostras de areia coletadas.

As escolas, do município I e do município II, que representam respectivamente a região no noroeste e interior do estado totalizou cem amostras para pesquisa e quaisquer não tiveram os seus nomes citados a fim de evitar possíveis constrangimentos. Os ambientes escolares, de um modo geral, eram limpos e organizados, oferecem um amplo espaço para a realização de atividades recreativas, sendo que todas os espaços de recreação foram observados solo composto de areia, grama e terra, as cercas eram baixas, com isso, a presença de animais eram constantes e observáveis. Os alunos matriculados variam de 3 meses a 5 anos de idade. Sendo que município I apresenta 22 escolas e o município II possui 6 escolas, entretanto a pesquisa tratou 5 escolas apenas de cada cidade. A etapa de coleta ocorreu durante os meses de março e maio de 2018. Em virtude, as localizações das escolas eram tanto em bairros como centro da cidade. Não participaram da pesquisa todas as escolas que não apresentavam locais de acesso público com areia, nem próximo às edificações. A amostra foi constituída por areia de pátios internos e dos entornos de escolas infantis da rede municipal de dois municípios do Estado do Rio Grande do Sul

Para análise parasitológica coletou-se dez amostras de areia, pesadas em balança digital portátil (10 kg × 1g SF400) com aproximadamente $150 \mathrm{~g}$, sendo cinco superficiais e cinco profundas

Tabela 1: Amostras positivas de parasitos encontrados nos dois municípios.

\begin{tabular}{lccc}
\hline Variáveis & $\begin{array}{c}\text { Total de } \\
\text { amostras } \\
\text { analisadas }\end{array}$ & $\mathbf{N}^{*}$ & $\%$ \\
\hline Município I & 50 & 27 & $54 \%$ \\
Município II & 50 & 28 & $56 \%$ \\
\hline
\end{tabular}

(com profundidade em média $10 \mathrm{~cm}$ ) em cada creche, totalizando 50 amostras por escolas. Foram de caráter randômico as amostras em locais sombreados e no sol. As amostras foram colhidas respeitando à equidistância de um metro de distância mínima e no máximo de dois metros de uma amostra para outra, assim, buscando evitar viés na seleção das amostras de solo. A areia foi coletada com uma pá de jardinagem, e para demarcar a profundidade foi utilizado uma régua de $30 \mathrm{~cm}$, e pinos coloridos para marcar os locais exatos das amostras. No momento da coleta das amostras de areia, foram observadas as características ambientais como natureza do solo, a temperatura ambiente e a umidade, presença de lixo e fezes de animais ${ }^{6,12}$. As sujidades presentes junto às amostras foram retiradas durante a própria técnica de isolamento parasitário.

Posteriormente, as amostras foram armazenadas em sacos plásticos lacrados e etiquetados (data/local), assim como também acompanhadas pela ficha individual descritiva da área escolar, sendo posteriormente transportas até o local das análises laboratoriais e mantidos em caixa térmica para transporte. O tempo entre a coleta e processamento foi de no máximo uma hora, durante esse intervalo, as amostras foram mantidas a $10^{\circ} \mathrm{C}$ em média. As porções de areia foram processadas, no laboratório de Histologia II da Universidade de Cruz Alta, através do Método de Rugai e Hoffman, Pons e Janer ${ }^{6}$ adaptada para utilizar amostras do solo. Após esse período foi descartado o sobrenadante e apenas o sedimento foi analisado e após confeccionadas duas lâminas para análise duplo cego em microscopia óptica visualizadas nas objetivas 10x e 40x.

Para analise estatísticas univariada, as variáveis qualitativas as tabelas de distribuição de frequências (n e \%). Para associação entre duas variáveis qualitativas, obtidas através do teste de Qui-Quadrado, com nível de significância $\alpha=5 \%$.

\section{RESULTADOS}

De um total de 100 amostras de areia coletadas do solo das áreas de recreação de dez creches municipais, $55 \%$ mostraram-se positivas para a espécie de parasito de homem ou de animais, variando pouco a intensidade de contaminação do solo entre as creches em relação aos municípios (Tabela 1).

Ao analisar as amostras das dez escolas de ambos os municípios, foi verificada a presença de ovos larvas do gênero Ancylostoma spp., entretanto, é necessário por ser semelhante ao parasito Strongyloides stercoralis distinguir da lavra rabditoide de Ancilostomídeos, ou seja, que por apresentar-se com vestíbulo bucal curto e primórdio genital bastante nítido confirmar-se lavras de Ancylostoma spp ${ }^{5}$. Na Tabela 2 estão descritos os 
Tabela 2: Frequência do parasito nas amostras de areia coletadas do município I e II, respectivamente.

\begin{tabular}{lccc}
\hline \multicolumn{1}{c}{ VARIÁVEIS } & AMOSTRAS & $\mathbf{N}^{*}$ & $\%^{*}$ \\
\hline MUNICIPIO I: & 50 & 27 & 54 \\
LOCALIZADO NO & & & \\
NOROESTE DO & & & \\
ESTADO. & 10 & 4 & 40 \\
ESCOLAA & 10 & 6 & 60 \\
ESCOLA B & 10 & 6 & 60 \\
ESCOLA C & 10 & 5 & 50 \\
ESCOLA D & 10 & 6 & 60 \\
ESCOLA E & 50 & 28 & 56 \\
MUNICÍPIO II: & & & \\
MUNICÍPIO & & & \\
LOCALIZADO NO & & & \\
INTERIOR DO ESTADO & 10 & 8 & 80 \\
ESCOLAA & 10 & 6 & 60 \\
ESCOLA B & 10 & 5 & 50 \\
ESCOLA C & 10 & 4 & 40 \\
ESCOLA D & 10 & 7 & 70 \\
ESCOLA E &
\end{tabular}

$\mathrm{N}^{*}$ e $\%^{*}=$ Número de amostra total e porcentagens positivas.

resultados encontrados em cada município, sendo que as escolas estão representadas pela letra do alfabeto ( $A, B, C, D$ e $E$ ), ressaltando que foram coletadas dez amostras de cada escola.

$\mathrm{Na}$ verificação da influência pela sobrevida de formas parasitárias observa pouca diferença na frequência de amostras positivas quando comparadas as profundidades (profundo e superfície) nas quais as amostras foram de $45 \%$ para o município I e município II $60 \%$ ambas da área profunda a presença de maior número de helmintos. Pode-se justificar a presença de maior número de helmintos em áreas profundas e sombreadas devido ao hábito dos animais enterrarem suas fezes após a eliminação dos seus dejetos, eliminando, portanto, formas infectantes que podem permanecer vários meses no solo. Assim, a contaminação das caixas de areia usadas para recreação infantil constitui grave problema de saúde pública em virtude da possibilidade de transmissão de parasitoses, onde estas são capazes de afetar o equilíbrio nutricional e gerar complicações no metabolismo normal intestinal da criança ${ }^{13-16}$.

\section{DISCUSSÃO}

Pesquisas que apresentam como finalidade avaliar a presença de estruturas pré-parasitárias (ovos e larvas) de helmintos ou protozoários em areias de áreas públicas, de recreações, ou seja, que tenha acesso tanto de animais quanto de pessoas, assim demonstram notoriedade em saúde pública por despertar sobre a possibilidade dos riscos de infecções eminentes a humanos, contribuindo para diminuição de possíveis casos. No presente estudo foi possível observar a presença de ovos e larvas do helminto nematódeo Ancylostoma spp. em areias de escolas municipais em dois municípios.

De acordo com World Health Organization $(W H O)^{17}$ as infecções parasitárias dependem de vários fatores, sendo um deles a exposição de indivíduos em determinados locais reconhecidos como potenciais focos de infecção. A contaminação do solo e da água por excretas humanas e animais faz com que a ocorrência de doenças parasitárias aumente principalmente em indivíduos que mantém um contato duradouro com tais locais ${ }^{18}$.

No período durante as coletas foi observado que cães e gatos tinham acesso aos locais, apesar de todas essas escolas apresentarem cercas ao seu redor, a explicação seria que cercas que apresentavam eram baixas, assim era possível que os animais podiam ter contato com o ambiente. Tal fato justifica a observação de ovos e larvas presentes nas amostras (55\% das amostras de ambos municípios), entretanto em relação ao município I, a pesquisa tratou de 22 , $7 \%$ das escolas e no município II foram pesquisadas mais da metade das escolas (83,3\%). Este resultado corrobora com os estudos prevalência de helmintos em areias de praças públicas ${ }^{4,5}$, que observaram a frequência positiva de larvas e ovos dos helmintos da família Ancylostomatidae em areias das creches pesquisadas. Além do estudo de Graciliano et al. ${ }^{14}$ que da mesma forma verificaram, em amostras de areia, a presença de larvas de Ancilostomídeos. Isto comprova a importância de pesquisas de cunho epidemiológico para a implantação de medidas de restrição ao acesso de animais em locais públicos como a instalação de cercas no entorno desses locais. Entretanto Mascaranhas e Silva ${ }^{8}$ atentam e reforça para a obrigatoriedade de cobrir os locais com areia nos períodos noturnos, sendo insuficiente a utilização das cercas.

No estudo Silva et al. ${ }^{19}$, atribuiu à ausência de ovos e de larvas de Toxocara spp. e Ancylostoma spp. no espaço escolar devido o difícil o acesso é de cães, sendo que frequente a presença de gatos costumam apresentar baixas infecções que confirma os dados obtidos na pesquisa que uma das causas é o acesso de cães no local. Outro dado relevante, demostrou que das amostras positivas a maior porcentagem era do ambiente "externo", totalizando $70 \%$ nos ambos municípios.

No entanto, devido as crianças utilizam como parte do fardamento sandálias, não havendo a obrigatoriedade de uso de sapato fechado, facilitando ainda mais a contaminação destas por Larva Migrans 
e outras parasitoses até mesmo devido a estação climática (verão) e o tempo médio de permanência das crianças nestas áreas era de 30 minutos diários.

Atualmente estudos com plantas tem sido cada vez mais revelante, Rey $^{9}$ cita que grande número de substâncias de origem vegetal é capaz de interferir na biologia de larvas, destruindo-a. Assim sendo, recomenda-se como plantio dos vegetais, em locais de focos, tais plantas: Cymbipogon citratos, C. martinii, Vetiveria zizanoides, Ruta graveolens, Menta spicata e Chrsanthemum sp. no controle antilarvario de Ancylostoma spp. no solo, assim como menciona a importância de medidas profiláticas ${ }^{5,9}$.

No presente estudo, tratam sobre a veiculação ambiental de formas infectantes de protozoários e helmintos, os quais constituem um grande problema de saúde pública, por afetarem diretamente a saúde do homem. Assim como neste estudo, que teve como objetivo verificar o grau de contaminação parasitológica de espaços públicos infantis e que comprovou a facilidade da infecção parasitária humana através do simples contato com o solo.
Considerando o grau de contaminação existente nos espaços físicos estudados, o resultado deste estudo aponta para uma situação que inspira cautela, por se tratar de locais públicos frequentados por crianças e adultos. Sendo assim, revela um resultado bem significativo sobre contaminação dos solos de parque de recreação das creches públicas por ovos e larvas de helmintos com potencial zoonótico. Onde os principais agentes contaminadores são os cães e gatos, sendo necessário uma conscientização dos profissionais de saúde pública, veterinários, diretores de creches e principalmente da população sobre os riscos e agravos da presença desses helmintos no ambiente, na população canina e humana.

\section{Agradecimentos}

Nosso agradecimento aos municípios, as escolas participantes da pesquisa e à Universidade de Cruz Alta.

\section{Conflitos de Interesse}

Os autores declaram não ter conflitos de interesse.

\section{REFERÊNCIAS}

1. Gurgel RQ, Cardoso GS, Silva ÂM, Santos LN, Oliveira RCV. Creche: ambiente expositor ou protetor nas infecções por parasitas intestinais em Aracaju, SE. Rev Soc Bras Med Trop. 2005;38(3):267-9. http://dx.doi.org/10.1590/S003786822005000300014

2. Martins RS, Alves VM. Análise de areias de parques públicos nos municípios de Castelo e Cachoeiro de Itapemirim, Espírito Santo. Pubvet. 2018;12(5):1-9. http://dx.doi. org/10.22256/pubvet.v12n5a81.1-9.

3. Andreis A, Schuh GM, Tavares RG. Contaminação do solo por parasitas e ocorrência. Estudos. 2008;12:1169-8.

4. Araújo NDS, Rodrigues CT, Cury MC. Helmintos em caixas de areia em creches da cidade de Uberlândia, Minas Gerais. Rev Saude Publica. 2008;42(1):1503. http://dx.doi.org/10.1590/ S0034-89102008000100021. PMid:18200354.

5. Maciel JS, Esteves RG, Souza MA. Prevalência de helmintos em areias de praças públicas do município de São Mateus, Espírito Santo, Brasil. Natureza on line. 2016;14:15-22.
6. Rugai E, Mattos T, Brisola AP. Nova técnica para isolara larvas de nematóides das fezes: modificações do Método de Baermann. Rev Inst Adolfo Lutz. 1954;14:5-8.

7. Araujo HB Fo, Carmo-Rodrigues MS, Mello CS, Melli LCFL, Tahan S, Morais MB. Parasitoses intestinais se associam a menores índices de peso e estatura em escolares de baixo estrato socioeconômico. Rev Paul Pediatr. 2011;29(4):521-8. http://dx.doi.org/10.1590/S010305822011000400009.

8. Mascaranhas JP, Silva DS. Presença de parasitos no solo das áreas de recreação em escolas de educação infantil. J Nutr Health. 2016;6(1):6-8.

9. Rey L. Parasitologia humana. 2. ed. Rio de Janeiro: Guanabara Koogan; 1991.

10. Santos B. Avaliação da contaminação por parasitas zoonóticos em praças públicas da cidade de Muriaé (MG). Rev Cient Faminas. 2017;12(2):46-51.

11. Sousa JO, Santos EO, Lira EM, Sá IC, Hirsch-Monteiro C. Análise parasitológica da areia das praias urbanas de João Pessoa/PB. Rev
Bras Cienc Saude. 2014;18(3):195-

7. http://dx.doi.org/10.4034/

RBCS.2014.18.03.02.

12. Neves D. Parasitologia humana. São Paulo: Atheneu; 2016. p. 115-4.

13. Araújo FR, Araújo $C P$, Werneck $M R$, Górski A. Larva migrans cutânea em crianças de uma escola em área do Centro-Oeste do Brasil. Rev Saúde. 2000;2(1):84-5. http://dx.doi.org/10.1590/S003489102000000100015.

14. Graciliano N, Farias JAC, Matos R. Contaminação de areia por parasitos de importância humana detectados nas praias da orla marítima de Maceió-AL. Arq Med Hosp Fac Ciên Méd St. Casa São Paulo. 2017;62:814.

15. Komagome SH, Romagnoli MPM, Previdelli ITS, Falavigna DLM, Dias MLGG. Fatores de risco para infecção parasitária intestinal em crianças e funcionários de creche. Cienc Cuid. 2007;6:442-7.

16. Nunes CM, Pena FC, Negrelli GB, Anjo CGS, Nakano MM, Stobbe NS. Ocorrência de larva migrans na areia de áreas de lazer das escolas municipais de ensino 
infantil, Araçatuba, SP, Brasil. Rev Saude Publica. 2000;34(6):656-8. http://dx.doi.org/10.1590/S003489102000000600015 . PMid:11175613.

17. Organização Mundial de Saúde. Infectious diseases of potential risk for travelers. WHO; 2018.
18. Magalhães TDR, Coelho MDG, Araújo AJUS, Coelho FAS. Influência de fatores socioambientais na ocorrência de enteroparasitos e protozoários não patogênicos em área periférica do município de Cristina, MG - Brasil. Rev Biociências. 2013;19(2):18-26.
19. Silva JP, Marzochi MCA, Santos ECL. Avaliação da contaminação experimental de areias de praias por enteroparasitoses: pesquisa de ovos de helmintos. Cad Saude Publica. 1991;7(1):90-9. http:// dx.doi.org/10.1590/S0102311X1991000100008.

Recebido: 8 ago, 2018 Aceito: $10 \mathrm{abr}, 2019$ 